\title{
Total internal reflection optical switch in SOI with defect engineered barrier region
}

\author{
D. J. Thomson, G. T. Reed, A. P. Knights, P. Y. Yang, F. Y. Gardes, A. J. Smith \& K. L. Litvinenko.
}

\begin{abstract}
Total internal reflection optical switches offer a switching operation which can be wavelength insensitive, thermally stable and polarisation independent. The implementation of such a switch based upon carrier injection in silicon is difficult due to the long diffusion lengths of injected free carriers. In this paper experimental results are presented which show that a reflective type switching operation is obtainable if a barrier formed of defective silicon is used to reduce free carrier diffusion.
\end{abstract}

Index Terms - Silicon Photonics, Optical Switch, Total Internal Reflection, Carrier Confinement, Defect engineering

\section{INTRODUCTION}

$\mathrm{O}$ ptical switches which are based upon Total Internal Reflection (TIR) can be advantageous over other switching topologies, in providing a compromise of the different performance metrics. Whilst MEMS based devices offer superior crosstalk than other switching types, they are slow, bulky, relatively complicated to fabricate and offer fewer opportunities for electronic-photonic integration. Mach Zehnder Interferometer (MZI), directional coupler (DC), ring resonator (RR), multimode interference (MMI) and TIR switching devices can be formed using CMOS compatible processes and both front and back end integration schemes are conceivable. Multi-GHz switching can be achieved in MZI and DC devices which are based within sub-micrometer waveguides as carrier depletion is possible, although such devices typically require millimetre interaction lengths as do MMI switches. RR devices can operate at equally high speeds, and also boast compactness ( $\sim 10$ 's $\mu \mathrm{m})$, but are highly sensitive to wavelength, temperature and fabrication tolerances. MMI and DC devices also suffer significant sensitivity to changes in temperature and wavelength. TIR devices typically require a moderate interaction length $(\sim 100$ 's $\mu \mathrm{m})$, are temperature and wavelength insensitive, and unlike other switching types can be overdriven without a pre-emphasis signal. However, the large refractive index change required to switch these devices, necessitates carrier injection; devices are therefore limited to $\sim \mathrm{MHz}$

Manuscript submitted January 12, 2010. This work was supported by the EPSRC.

D. J. Thomson, G. T. Reed, P. Y. Yang, F. Y. Gardes, A. J. Smith and K. L. Litvinenko are with the Advanced Technology Institute, University of Surrey, Guildford, Surrey, GU2 7XH, UK. (d.thomson@surrey.ac.uk)

A. P. Knights is with the Department of Engineering Physics, McMaster University, Hamilton, Ontario, L8S 4L7, Canada. operation, limiting the applications for which they are useful, but enabling efficient devices to be fabricated. Careful design of each of the switching types including the TIR switch can theoretically allow polarisation independent operation. The TIR switch offers a balance of the different performance metrics and could therefore be attractive for use in the circuit switching layer of a futuristic switching network where the disadvantages of the MEMS switch become problematic.

The switching operation is achieved in a total internal reflection switch by creating a controllable refractive index change in the device such that an interface between two regions of unequal refractive index exists which causes a reflection. Controllable refractive index changes can be achieved in silicon through the use of the plasma-dispersion or thermo-optic effects. Reflection type devices which use the thermo-optic effect have been reported in silicon [1], however the main draw back is the relatively slow switching rate due to the time required to generate and dissipate heat.

Plasma dispersion based reflective switches which incorporate a $p-i-n$ diode structure to inject free carriers into the device have also been reported in both silicon [2-6] and other semiconductor materials [7-10]. In order to produce an efficient reflection, a sufficiently large and abrupt spatial variation in refractive index is required during injection. This is difficult to achieve in silicon where the diffusion lengths of injected carriers are relatively long. This becomes especially apparent as device dimensions are reduced towards sub-micron, such has been the trend in silicon photonics. The importance of having a precise controllable reflection interface in this type of switch is widely acknowledged [3-6, 10] and problems caused by the high injection currents required for switching have also been discussed [7].

Attempts have been made to confine the injected carriers to certain regions within the device during switching in devices fabricated in materials other than silicon [7, 9, 11, 12]. Whilst these devices have all reported an improved performance, none of the designs show any method of lateral carrier restriction in the guiding layer itself and therefore carriers are still able to diffuse through the guiding layer freely, compromising device operation.

Defect engineering has emerged as an exciting prospect in silicon photonics with proposed use in the development of optical detectors [13], amplifiers [14] and emission devices [15]. Previously we showed the feasibility of using a thin $\mathrm{SiO}_{2}$ barrier to prevent carrier diffusion in reflective switches [16]. In this work we utilize the electrical properties of defective silicon to create a barrier to carrier diffusion in the TIR switch and as a result improve the magnitude and abruptness of the spatial refractive index change during the switched state. 
Experimental results obtained from fabricated devices are presented which demonstrate how a switching operation can be achieved through the incorporation of the barrier. This is in contrast to the results also presented from the devices fabricated without barriers, where no switching was observed.

\section{SWITCH DESIGN AND OPERATION}

The layout of the switch is shown in figures $1 \mathrm{a}$ and $1 \mathrm{~b}$. Two waveguides of height $1.5 \mu \mathrm{m}$, slab height $0.7 \mu \mathrm{m}$ and starting width $1 \mu \mathrm{m}$ are defined to intercept at an angle $\theta$. These rib waveguide dimensions are chosen to give single mode propagation. The waveguides are tapered to a width of $6 \mu \mathrm{m}$ as they approach the interception region in order to reduce crosstalk. A shallow $p^{+}$doped region is located along the top of the reflection interface and an $n^{+}$doped region exists in the slab, with ohmic connections to an anode and cathode respectively. These doped regions are separated by intrinsic single crystal silicon to therefore form a $p-i-n$ diode structure on one side of the reflection interface. On the other side of the interception region to the diode structure is a region of defective silicon which is used as the barrier to reduce carrier diffusion. The length of the active region ranges from $195 \mu \mathrm{m}$ to $113 \mu \mathrm{m}$ for devices with interception angles $1.5^{\circ}$ to $3^{\circ}$, or $500 \mu \mathrm{m}$ if the tapers which approach the interception region are included. It is expected that optimisation of the taper shape can result in significant overall device length reductions.

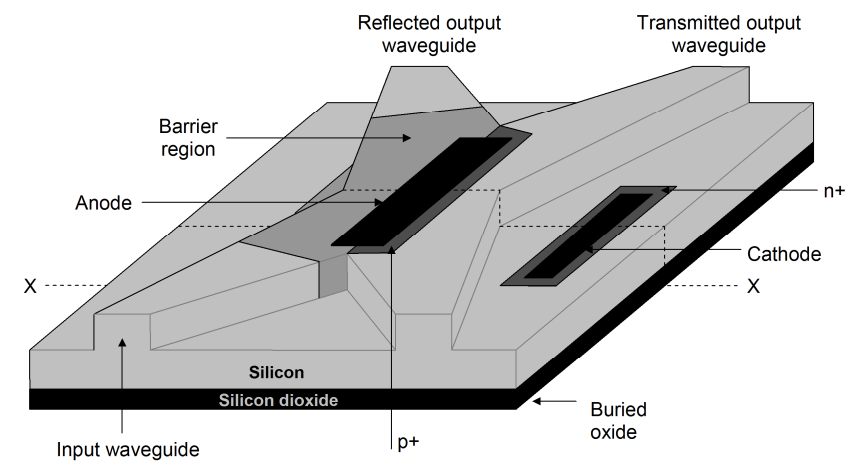

Fig. 1a. Diagram of device layout.

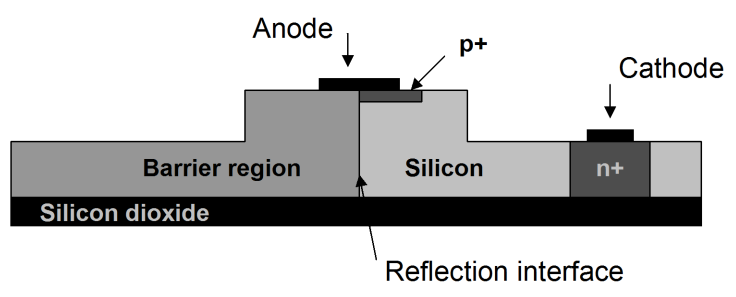

Fig. 1b. Cross sectional view along line XX.

With no current passing through the diode the input light propagates along the input waveguide and passes through the 'reflection interface' to the 'transmitted' output waveguide. When the device is forward biased free carriers are injected mainly into the intrinsic silicon region to the right hand side of the reflection interface. These injected carriers cause a reduction in refractive index due to the free carrier plasma dispersion effect [17]. If a sufficient refractive index change occurs the input light will be incident on the reflection interface at an angle greater than the critical angle for total internal reflection meaning that the input light will be switched to the 'reflected' output waveguide and thus switching occurs.

\section{DEFECT BARRIER DESIGN}

The defective silicon barrier region was formed using ion implantation. One major advantage of this method is the ability to selectively damage certain regions by depositing and patterning masking material capable of blocking the implant. $\mathrm{Si}^{+}$ ions were used for the implantation in order to avoid any chemical effects which might result from using other elements. An implantation recipe was selected which caused full amorphisation of the unmasked overlayer. Once amorphised, a thermal process was performed in order to recover the optical properties to some extent. Complete amorphisation of the silicon overlayer removes any seeds which are required for epitaxial growth (other than a small contribution from lateral regrowth). During the thermal process random nucleation occurs instead which results in the formation of polycrystalline silicon.

The free carrier lifetime and refractive index of the resulting polycrystalline silicon are critical to the device operation. The refractive index of the defective silicon regions should not be significantly different to that of single crystal silicon such that the input light is reflected without carrier injection. The refractive index of polycrystalline silicon for a wavelength of $1550 \mathrm{~nm}$ formed using ion implantation and annealing at different temperatures has been experimentally characterised using ellipsometry [18], the results of which are shown in figure 2

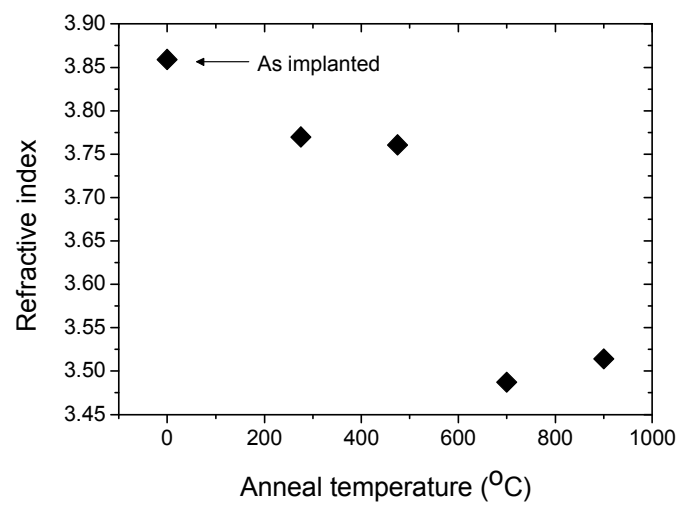

Fig. 2. Refractive index of amorphised and thermally regrown poly crystalline silicon.

The refractive index of the as-implanted sample is approximately 0.4 higher than that of single crystal silicon. This is consistent with previous measurements of ion implantation amorphised samples [19]. The samples annealed at temperatures up to $500^{\circ} \mathrm{C}$ have a slightly lower refractive index than that of the as implanted sample. The samples annealed at $700^{\circ} \mathrm{C}$ degrees and above have a refractive index recovered to approximately that of single crystal silicon as required in the 
switch. To analyse the importance of the free carrier lifetime in the barrier region, the effectiveness of the barrier at improving the abruptness and magnitude of the spatial injected free carrier profile across the intended reflection interface was modelled using the commercial software ATLAS [20].

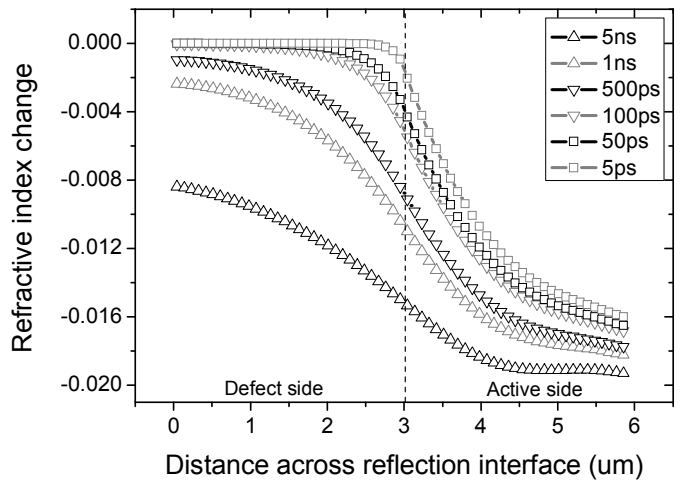

Fig. 3. Refractive index profiles produced in the switching region during carrier injection with different free carrier lifetimes in the barrier region.

Devices were modelled with the barrier region defined with different free carrier lifetimes. A $1.2 \mathrm{~V}$ forward bias was applied to the device and the profile of free carriers across the reflection interface was extracted and converted to refractive index change using Eqn. (1) following the work by Soref and Bennett [17]. The results of this modelling are shown in figure 3.

$$
\Delta n=\Delta n_{e}+\Delta n_{h}=-\left[\left(8.8 \times 10^{-22} \Delta N_{e}\right)+\left(8.5 \times 10^{-18}\left(\Delta N_{h}\right)^{0.8}\right)\right]
$$

A free carrier lifetime of $5 \mathrm{~ns}$ was measured for the intrinsic waveguides used in this work through an optical pump-probe method. As the free carrier lifetime of the barrier region is reduced below $5 \mathrm{~ns}$ both the abruptness and magnitude of the spatial refractive index variation across the reflection interface (position indicated by dashed line) are enhanced. To analyse how this enhancement improves the switching performance of the device, the refractive index profiles shown in figure 3 have been imported into a BPM optical modelling package from RSoft [21].

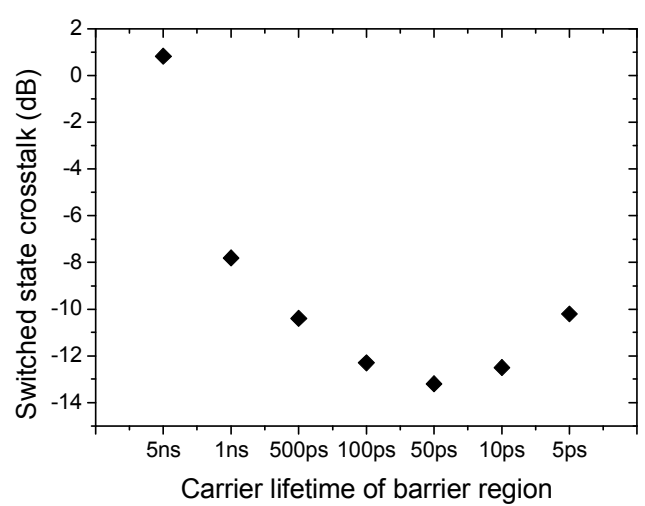

Fig. 4. Switched state crosstalk with different barrier region free carrier lifetimes.
The output powers from the reflected and transmitted output waveguides were monitored for each refractive index profile and converted to switched state crosstalk using Eqn. (2):

$$
X_{s}=10 L O G\left(\frac{P_{t}}{P_{r}}\right)
$$

where $P_{t}$ is the power from the transmitted output waveguide and $P_{r}$ is the power from the reflected output waveguide. The modelled crosstalk results for each barrier region free carrier lifetime are shown in figure 4.

A low crosstalk value indicates that the majority of the output power is exiting the device from the reflected output waveguide as is required in the switched state. According to the model, optimal crosstalk is obtained if the barrier region has a free carrier lifetime of approximately 50ps. With a free carrier lifetime of $50 \mathrm{ps}$ the free carrier concentration in the barrier region has already been minimised. The abruptness will improve further as the lifetime is reduced below 50ps however; this itself increases the diffusion flux of free carriers from the active region to the barrier region causing a reduction in the magnitude of the refractive index change.

The free carrier lifetime of SOI, amorphised and thermally regrown at different anneal temperatures, has been experimentally examined using a reflective optical pump-probe method. Carriers are generated in the overlayer using a high intensity pump beam. A probe beam is then made incident on the same spot and the reflected power of this beam monitored. As carriers are generated in the overlayer the reflected power is reduced. After the pump beam is switched off, these carriers will recombine without the generation of new carriers, increasing the reflected power at a rate dictated by the material's free carrier lifetime. The results from this experiment are shown in figure 5 . An extremely long decay is observed in the unimplanted single crystal silicon sample. The as-implanted (amorphous) sample has a very rapid decay. The decay rate tends to then reduce with increasing anneal temperature; however the 1/e free carrier decay level is reached within $50 \mathrm{ps}$ for all considered anneal temperatures.

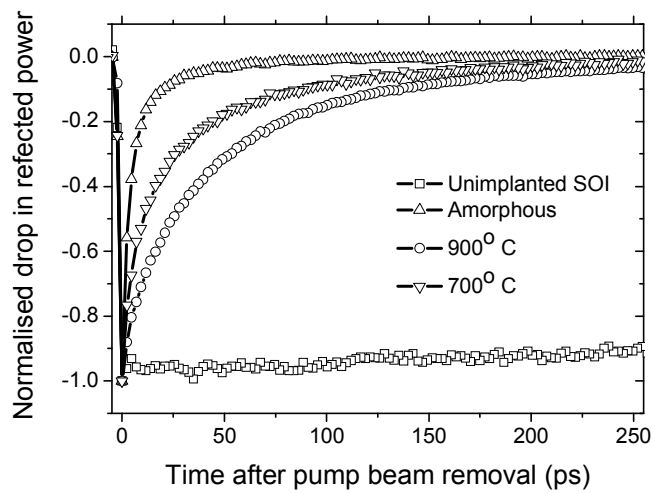

Fig. 5. The time dependent decay of optically generated free carriers in amorphised and thermally regrown poly crystalline silicon after the removal of the pump beam. 


\section{DEVICE FABRICATION}

Devices were fabricated in the cleanroom facilities at the University of Surrey. The starting material was (100) SOI with a $1.5 \mu \mathrm{m}$ thick overlayer and $2 \mu \mathrm{m}$ thick buried oxide layer. The implantation steps were performed first, commencing with the highly doped regions. The $p^{+}$region was formed by implanting a $2 \mathrm{e} 15 \mathrm{ion} / \mathrm{cm}^{2}$ dose of boron at an energy of $5 \mathrm{keV}$. A $1 \mathrm{e} 16$ ions $/ \mathrm{cm}^{2}$ dose of phosphorus at an energy of $640 \mathrm{keV}$ was implanted to produce the $n^{+}$region. An anneal was then performed at $1050^{\circ} \mathrm{C}$ for 10 seconds to activate the doping and to repair any implant damage. The barrier region was then formed using a chain implant of energies $1400 \mathrm{keV}, 900 \mathrm{keV}$ and $500 \mathrm{keV}$ at doses of $5 \mathrm{e} 15,3.5 \mathrm{e} 15$ and $3 \mathrm{e} 15 \mathrm{ions} / \mathrm{cm}^{2}$ respectively. In order to minimise the effect of self annealing during the implantation the sample stage was cooled with liquid nitrogen. After implantation the samples were annealed at $800^{\circ} \mathrm{C}$ for 10 minutes. This recipe was shown in the previous section to produce polycrystalline silicon with suitable optical and electrical properties. To ensure that the implantation recipe had left no pockets of single crystal material which could act as a seed for solid phase epitaxial regrowth, SOI samples from the same wafer as the device samples were implanted and annealed with the same recipe as used in the devices. Rutherford back scattering in the channelling configuration was then performed (results not shown). An insignificant degree of ion channelling in the regrown sample was observed indicating the presence of significant lattice disorder. The actual shape and position of the barrier region edge deviates from the ideal case as depicted in figure $1 \mathrm{~b}$ due to the shape of the implantation mask edge, and ion implantation effects. This deviation has been modelled and shown to not have a significant effect on the free carrier profile produced with the device under forward bias.

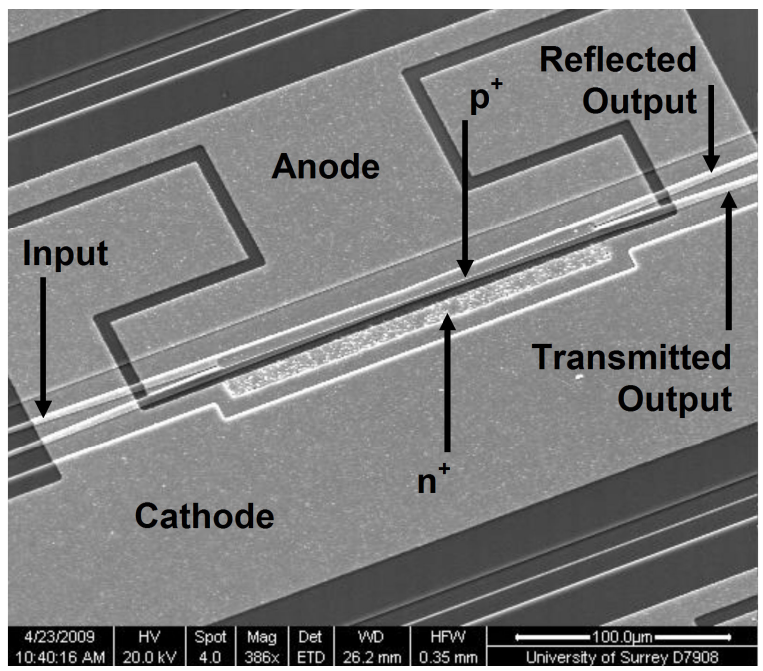

Fig. 6. SEM image of a fabricated device.

After the implantation and anneal steps were completed the waveguides were etched to a nominal depth of $800 \mathrm{~nm}$. The etch depth was subsequently measured by Scanning Electron Microscopy (SEM) to be $720 \mathrm{~nm}$. A $500 \mathrm{~nm}$ thick $\mathrm{SiO}_{2}$ upper cladding layer was deposited using Plasma Enhanced Chemical Vapour Deposition and contact holes etched through to the doped regions using buffered hydrofluoric acid. A 500nm thick aluminium layer was deposited and selectively etched to form the electrodes. An annotated SEM image of an example fabricated device is shown in figure 6. After fabrication the device samples were diced and edges polished to ensure that the waveguide facets were optically smooth.

\section{EXPERIMENTAL RESULTS}

Laser light of wavelength $1550 \mathrm{~nm}$ and TE polarization was free space butt coupled into the waveguide input. The output light was captured using an optical detector. The passive waveguide loss was measured by the cut back method to be $12 \mathrm{~dB} / \mathrm{cm}$. The other contributions to device loss were also measured using cut back loss test structures. The optical loss of waveguides formed in barrier material was measured to be $44 \mathrm{~dB} / \mathrm{cm}$ and the loss due to the inclusion of the highly doped boron region at the top of the rib waveguide $27 \mathrm{~dB} / \mathrm{cm}$. The loss caused by metal in close proximity to the waveguide but separated by the upper $\mathrm{SiO}_{2}$ cladding layer was found to be $5 \mathrm{~dB} / \mathrm{cm}$. Since the length of waveguide for which the barrier, metal and $p$-type doped regions exist is only approximately $150 \mu \mathrm{m}$ in the devices, these losses equate to approximately $1.5 \mathrm{~dB}$.

A range of devices with different interception angles were produced $\left(1.5^{\circ}, 1.75^{\circ}, 2^{\circ}, 2.25^{\circ}, 2.5^{\circ}, 2.75^{\circ}\right.$ and $\left.3^{\circ}\right)$. The output power from the reflected and transmitted waveguides was measured with light coupled into the input waveguide and no bias applied to the diode. The experimental results (plotted points) obtained from the devices without barrier regions are shown in figure 7 together with the results of optical modelling (solid lines). A good agreement between the modelling and the experimental results is observed. For all measurable interception angles these devices behave as required, in that with no applied bias the majority of the power is output from the transmitted output waveguide.

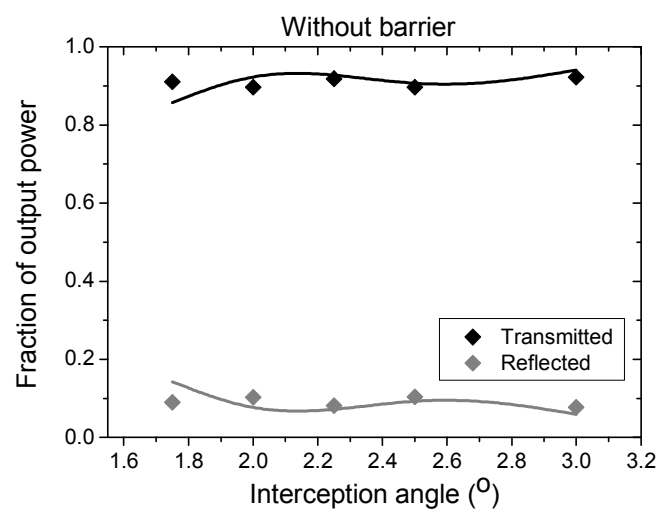

Fig. 7. Passive optical output powers from the devices without barrier regions.

Contrasting experimental results were obtained from the devices with barriers as is shown in figure 8 . For the devices with the two smallest interception angles $\left(1.5^{\circ}\right.$ and $\left.1.75^{\circ}\right)$ the majority of the power leaves the device from the reflected output waveguide. For the devices with interception angles $2^{\circ}$ and above the majority of the power is output from the transmitted output waveguide. This difference in behaviour 
between the devices with and without barrier regions can be attributed to the barrier region having a larger refractive index than that of the surrounding single crystal silicon. At the interface of the barrier region and the surrounding silicon some degree of reflection will be caused, the magnitude of which is determined by the difference in refractive index of the two regions and the angle of interception. Since the input light is incident on the reflection interface at a larger angle in the devices with smaller interception angles, they are more sensitive to refractive index mismatch.

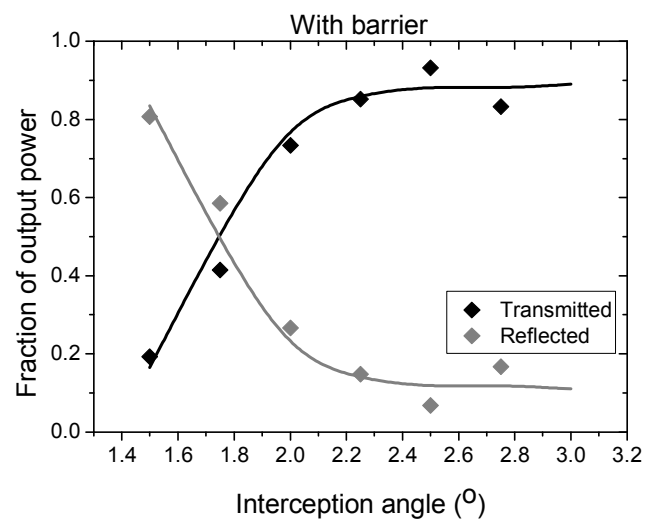

Fig. 8. Passive optical output powers from the devices with barrier regions.

This explains why the largest deviation in device performance between the devices with and without barriers is observed for the devices with the three smallest interception angles. Optical modelling has also been performed on the devices with different magnitudes of refractive index mismatch defined between the barrier region and the surrounding silicon. When a mismatch in refractive index of 0.005 is assumed a good agreement with the experimental results can be observed, as can be seen in figure 8 (solid lines). The presence of this refractive index mismatch is supported further by the results obtained with forward bias applied to the devices. The results obtained from the device with a barrier region and a $1.5^{\circ}$ interception angle are shown in figure 9.

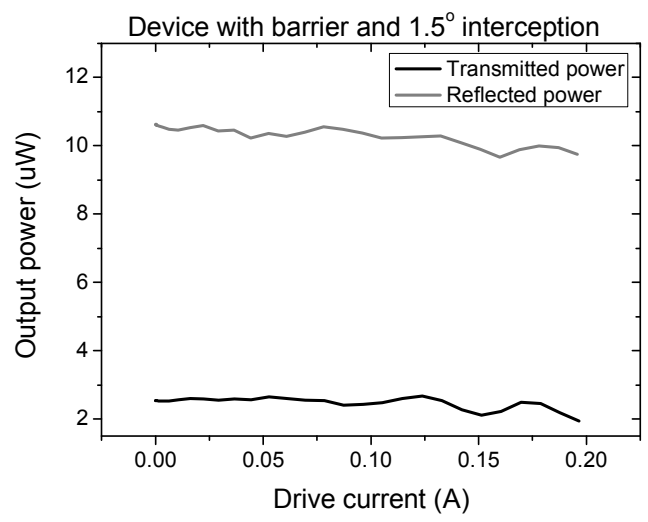

Fig. 9. Output power with increasing drive current for device with barrier and $1.5^{\circ}$ interception angle.
Consistent with figure 8 , with no applied bias the majority of the output power exits the device from the reflected output waveguide. As the drive current is increased any change in output powers is insignificant. In this case the refractive index mismatch alone is large enough to cause total internal reflection. Increasing the refractive index mismatch further by injecting free carriers into the device does not increase the reflected power since total internal reflection is already achieved.

This is in contrast to the results obtained from the devices with a $2^{\circ}$ interception angle shown in figures 10 and 11 . In the device without a barrier, with no current passing through the diode the majority of the power leaves the device from the transmitted output waveguide. When the drive current is increased the output power from the transmitted output waveguide decreases however this is not coupled with an increase in optical power from the reflected output waveguide. This device behaviour, which was also observed in all devices without barriers, can be attributed to the injected free carriers freely diffusing and causing absorption of the input light.

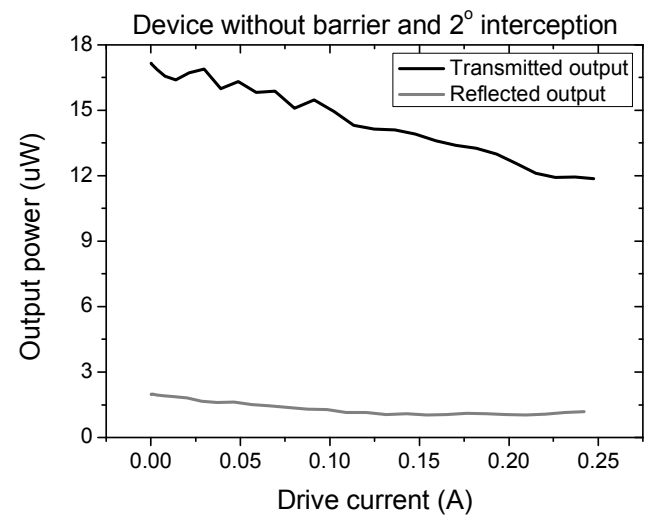

Fig. 10. Output power with increasing drive current for device without barrier and $2^{\circ}$ interception angle.

However, results consistent with the desired switching effect were obtained from the device with the inclusion of a carrier restrictive barrier as shown in Figure 11.

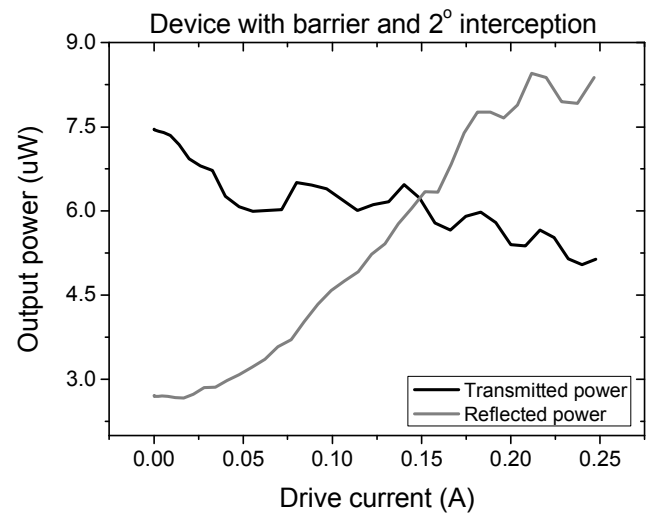

Fig. 11. Output power with increasing drive current for device with barrier and $2^{\circ}$ interception angle. 
With no applied bias, the majority of the output power exits the device from the transmitted output waveguide. When the drive current is increased the power from the transmitted output waveguide decreases, whilst the power from the reflected output waveguide increases such that there is a switch over in majority output power from the transmitted to reflected output. The switch over in optical power occurs with a switching current and corresponding power of approximately $150 \mathrm{~mA}$ and $440 \mathrm{~mW}$ respectively. In all of the devices with barriers and with an interception angle $>1.5^{\circ}$ an increase in reflected power and decrease in transmitted power was observed with increasing drive current. These results show the requirement of a barrier to restrict carrier diffusion in this type of switch and also that the use of defective silicon barrier is a feasible method of producing a switching operation in such a structure.

\section{Insensitivity of the switch to thermal ambient}

The switching operation of the device with barrier has also been analysed at an elevated temperature. The results obtained at $20^{\circ} \mathrm{C}$ and $70^{\circ} \mathrm{C}$ are shown in figure 12 .

The switching operation is almost identical at both temperatures indicating a high degree of thermal insensitivity as would be expected from a switch based upon reflection. This also demonstrates that the switching operation observed is being caused due to the presence of injected free carriers rather than heating of the device.

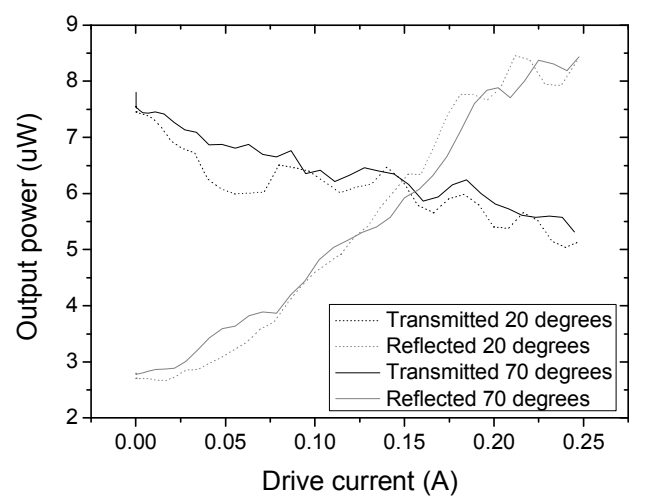

Fig. 12. Switching operation of device with barrier and $2^{\circ}$ interception angle at $20^{\circ} \mathrm{C}$ and $70^{\circ} \mathrm{C}$.

\section{Wavelength sensitivity of the switch:}

The wavelength sensitivity of the device has been analysed by measuring the switching operation at 3 different wavelengths spanning the maximum range of the laser $(1525 \mathrm{~nm}, 1550 \mathrm{~nm}$ and $1600 \mathrm{~nm})$ as shown in figure 13 . The switching operation observed for all three wavelengths is almost identical, highlighting again another advantageous property of this type of switch, in its wavelength insensitivity.

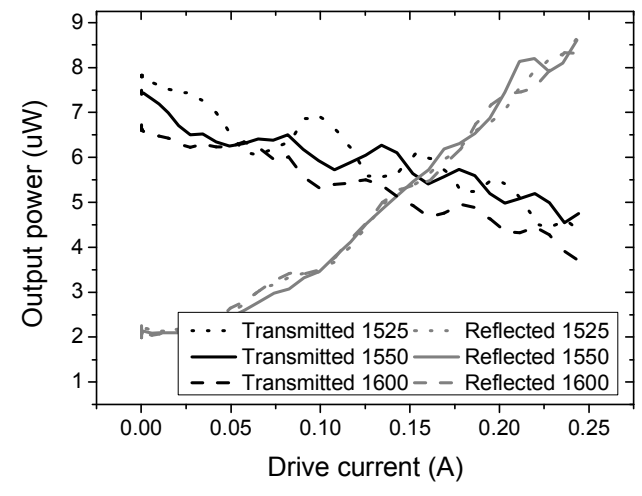

Fig. 13. Switching operation of device with barrier and $2^{\circ}$ interception angle at 1525,1550 and $1600 \mathrm{~nm}$.

\section{Switching time}

The response time of the switch has been investigated by analysing the optical output with the application of a $16 \mathrm{MHz}$ square wave signal across the diode. Rise and fall times of approximately $15 \mathrm{~ns}$ and $10 \mathrm{~ns}$ were measured respectively. To some extent the length of the measured switching times is a result of the finite transition times of the source which is operating at its upper frequency limit, therefore shorter switching times are expected.

The dynamic response of the device has also been modelled to give a further estimation of the switching speed. Modelling indicates that the device is limited by a rise time of $5 \mathrm{~ns}$ which corresponds to a switching bandwidth of $70 \mathrm{MHz}$.

Heat generation during the carrier injection process can be detrimental to the device operation due to the large and opposing thermal coefficient of silicon. In figures 3 and 4 it was demonstrated that for switching to occur, the refractive index mismatch must be sufficiently abrupt. At DC, heat generated in the PIN diode can rapidly dissipate throughout the overlayer and contacts to stabilise over a large area of the device. As a result no significant thermal gradients are expected in the interaction region and as was shown in figure 12, this type of global heating does not have a significant effect on the switching performance. At high speed, the relatively long heat generation and dissipation times generally limit switching times to $\mu \mathrm{s}$ whereas in this device, ns transitions have been observed. Free carrier based switching can therefore be concluded.

\section{Switching mechanism}

The configuration of switches based upon reflection, multimode interference and evanescent coupling is similar. The main structural difference is the interaction length as can be seen comparing previous devices reported in the literature $[6,22$, 23]. It is therefore important to confirm that the switching operation observed here is occurring due to reflection. Optical modelling has been performed on two slightly different structures configured as a multimode interference switch or a 
zero-gap directional coupler switch. The structures in the model have been configured with similar dimensions to those used for the switch described in this work. The devices were modelled with a global refractive index variation of -0.025 applied to the interaction regions. A refractive index change of this magnitude corresponds to temperature change of approximately $-160^{\circ} \mathrm{K}$ or an injected free carrier concentration of approximately $1.4 \times 10^{19} \mathrm{~cm}^{-3}$, both of which can be considered relatively large. For the multimode interference based device, the variation in output power from the two ports is very weak over the entire range. Some variation in the output powers from the coupler based device is observed, however it is small especially considering the magnitude of the refractive index change.

These findings are in good agreement with the devices reported in the literature where those based upon coupling or multimode interference require much greater interaction length than that of reflection based switches $[6,22,23]$. Further confirmation that the switching operation is not occurring due to a coupling type effect can be found when the wavelength sensitivity of such a device is observed. Both the coupler and the multimode interference configured devices were modelled for wavelengths in the range of $1520 \mathrm{~nm}-1600 \mathrm{~nm}$.

The device described in this work has been shown experimentally to be wavelength insensitive in this wavelength range; modelling indicates that this is not the case for the coupler based device. Only weak sensitivity is observed in the multimode interference based device. Another indication that the switching operation is occurring due to a reflective effect rather than a coupling or multimode interference based effect in response to a global refractive index change is that a switching operation would be expected from the devices without carrier restrictive barriers, which has been shown to not be the case. It can therefore be concluded that the switching operation observed in the device is occurring due to reflection.

\section{PROSPECTS FOR FUTURE DEVELOPMENTS}

The devices of this work were designed in order to prove the concept of the use of defective silicon barriers to improve device performance. In this respect successful results were obtained. For the device to be considered for practical applications it is appreciated that further performance enhancements are required. It is expected that through development of the waveguide formation process the passive optical waveguide loss and therefore the insertion loss of the device can be significantly reduced since waveguides of similar dimensions have been demonstrated with losses as low as $0.25 \mathrm{~dB} / \mathrm{cm}$ [24]. The crosstalk can be improved through the use of higher resolution lithography, and by considering the use of curved waveguide tapers on the approach to the switching region as is shown in optical modelling results of figure 14 .

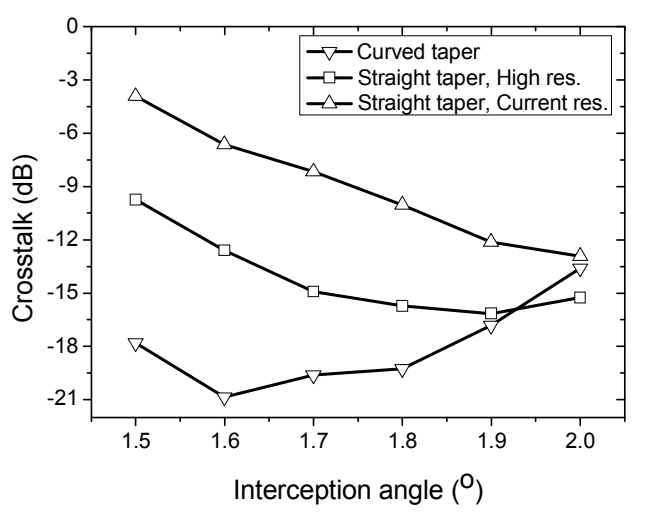

Fig. 14. Modelled crosstalk with current lithography, high resolution lithography and curved tapers.

Improvements in the switching efficiency, which will allow for a reduced drive current and an increased extinction ratio, can be obtained with greater control of the refractive index of the barrier region. Devices with smaller interception angles have a greater switching efficiency as is shown in the optical modelling results of figure 15 .

Switching efficiency in this device has been defined as the rate of change of crosstalk with varying refractive index mismatch. Removal of the passive refractive mismatch will allow for smaller angled devices to be used which will therefore have a greater switching efficiency. If the refractive index of the barrier region cannot be reduced by varying the process conditions alone, light $p$ or $n$-type doping can also be introduced for refractive index tuning.

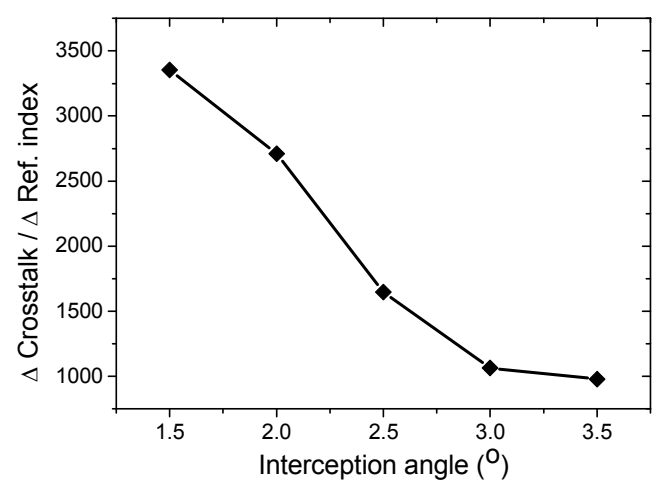

Fig. 15. Modelled efficiency of the device against interception angle.

Finally, the efficiency of the switching operation may be further enhanced by ensuring that end of range defects that are produced at the mask edge of the barrier region implant are not situated in the active region of the device. Instead a broader implant window may be defined and a longer thermal process used to regrow single crystal silicon laterally until the interface of the polycrystalline silicon (barrier region) and the single crystal silicon is situated along the middle of the interception region. The end of range defects can therefore be situated outside of the device area rather than in the interception region. 


\section{CONCLUSION}

In this paper the concept of the total internal reflection based optical switch in silicon with carrier restrictive barrier formed of defective silicon has been introduced. The advantages of this switching topology are wavelength insensitivity, thermal stability, polarization independence and the ability for overdriving without a pre-emphasis signal.

The results obtained from fabricated devices have demonstrated that without the use of a barrier a switching operation is not achievable. Instead, some absorption from the injected free carriers results with no increase in reflected power. When the barrier is introduced into the device, a switching operation can be observed. The switching operation of the device has been experimentally shown to be thermally stable and wavelength insensitive. It has also been proven to be occurring due to a reflective type operation in response to injected carriers rather than temperature change. The concept of the use of a defect silicon barrier has therefore been demonstrated. Further device developments have been described which can potentially result in a high performance optical switch in silicon.

\section{REFERENCES}

[1] F. Cantore and F. G. Della Corte, "1.55-m silicon-based reflection-type waveguide-integrated thermo-optic switch," Optical Engineering, vol. 42, pp. 2835-2840, 2003.

[2] Y. Gao, "SiGe/Si asymmetric $2 \times 2$ electro-optical switch of total internal reflection type," App. Phys. Lett., vol. 67, pp. 3379-3379, 1995.

[3] B. Li, G. Li, E. Liu, Z. Jiang, C. Pei, and X. Wang, " $1.55 \mu \mathrm{m}$ reflection-type optical waveguide switch based on $\mathrm{SiGe} / \mathrm{Si}$ plasma dispersion effect," App. Phys. Lett., vol. 75, pp. 1-3, 1999.

[4] Y. Liu, E. Liu, G. Li, S. Zhang, J. Luo, F. Zhou, M. Cheng, B. Li and H. Ge, "Novel silicon waveguide switch based on total internal reflection," App. Phys. Lett., vol. 64, pp. 2079-2080, 1994.

[5] B. Li and S. J. Chua, " $2 \times 2$ Optical waveguide switch with bow-tie electrode based on carrier-injection total internal reflection in SiGe alloy," IEEE Photon. Technol. Lett., vol. 13, pp. 206-208, 2001.

[6] C. Z. Zhao, A. H. Chen, E. K. Liu, and G. Z. Li, "Silicon-on-insulator asymmetric optical switch based on total internal reflection," IEEE Photon. Technol. Lett., vol. 9, pp. 1113-1115, 1997.

[7] Z. Wanru, D. Jining, Z. Zhengzhong, Y. Peisheng, S. Zhiwen, S. Furong, and G. Junhua, "Total internal reflection optical switch with injection region isolated by oxygen ion implantation," Fiber Integr. Opt., vol. 15, pp. 27-36, 1996.

[8] F. Ito, M. Matsuura, and T. Tanifuji, "A carrier injection type optical switch in GaAs using free carrier plasma dispersion with wavelength range from 1.06 to $1.55 \mu \mathrm{m}$," IEEE J. Quant. Electron., vol. 25 pp. 1677-81, 1989.

[9] K. Ishida, H. Nakamura, H. Matsumura, T. Kadoi, and H. Inoue, "InGaAsP/InP optical switches using carrier induced refractive index change," App. Phys. Lett., vol. 50, pp. 141-2, 1987.

[10] O. Mikami and H. Nakagome, "Waveguided optical switch in InGaAs/InP using free-carrier plasma dispersion," Electron. Lett., vol. 20, pp. 228-9, 1984.
[11] O. Kwang-Ryong, P. Ki-Sung, O. Dae-Kon, K. Hong-Man, P. Hyung Moo, and L. KwyRo, "A very low operation current InGaAsP/InP total internal reflection optical switch using $\mathrm{p} / \mathrm{n} / \mathrm{p} / \mathrm{n}$ current blocking layers," IEEE Photon. Technol. Lett., vol. 6, pp. 65-7, 1994.

[12] K. Wakao, K. Nakai, M. Kino, and S. Yamakoshi, "InGaAsP/InP optical switches embedded with semi-insulating InP current blocking layers." IEEE J. Sel. Areas Comm., vol. 6, pp. 1199-204, 1988.

[13] J. D. B. Bradley, P.E. Jessop, and A.P. Knights, "Silicon waveguide-integrated optical power monitor with enhanced sensitivity at $1550 \mathrm{~nm}$, , App. Phys. Lett., vol. 86, pp. 1-3, 2005.

[14] N. M. Wright, D. J. Thomson, K. L. Litvineko, W. R. Headley, A. J. Smith, A. P. Knights, J. H. B. Deane, F. Y. Gardes, G. Z. Mashanovich, R. Gwilliam, and G. T. Reed, "Free carrier lifetime modification for silicon waveguide based devices," Optics Express, vol. 16, p. 19779-19784, 2008.

[15] M. A. Lourenco, M.A., et al., M. Milosavljevic, G. Shao, R. M. Gwilliam, and K. P. Homewood, "Dislocation engineered silicon light emitting devices," Thin Solid Films, vol. 515, pp. 8113-17, 2007.

[16] D. Thomson, F. Y. Gardes, G. Z. Mashanovich, A. P. Knights and G. T. Reed, "Using $\mathrm{SiO}_{2}$ carrier confinement in total internal reflection optical switches to restrict carrier diffusion in the guiding layer. J. Lightw. Technol., vol. 26, pp. 1288-94, 2008.

[17] R. A. Soref and B. R. Bennett, "Electrooptical Effects in Silicon," IEEE J. Quant. Electron., vol. 23, p. 123-129. 1987.

[18] A. P. Knights, K. J. Dudeck, W. D. Walters, and P. G. Coleman, "Modification of silicon waveguide structures using ion implantation induced defects," Applied Surface Science, vol. 255, pp. 75-77, 2008.

[19] L. Pelaz, L. A. Marques, and J. Barbolla, "Ion-beam-induced amorphization and recrystallization in silicon," Journal of Applied Physics, vol. 96, pp. 5947-76, 2004.

[20] SILVACO International, 4701 Patrick Henry Drive, Bldg 1, Sant Clara, California 94054, USA. www.silvaco.com.

[21] Rsoft Design Group, 200 Executive Group Blvd. Ossining, NY 10562, USA. www.rsoftdesign.com.

[22] J. P. Lorenzo and R. A. Soref, " $1.3 \mu \mathrm{m}$ electro-optic silicon switch," App. Phys. Lett., vol. 51, p. 6-8, 1987.

[23] R. A. Forber and E. Marom, "Symmetric directional coupler switches" IEEE Journal of Quantum Electronics, vol. 22. pp. 911-19, 1986.

[24] V. Sih, X. Shengbo, K. Ying-Hao, R. Haisheng, M. Paniccia, O. Cohen and O. Raday, "Raman amplification of $40 \mathrm{~Gb} / \mathrm{s}$ data in low-loss silicon waveguides," Optics Express, vol. 15, 2007. 